Reseñas de Libros / Book and Report Review 


\title{
The practice of eating. Alan Warde. Cambrigde: Polity Press, 2016
}

\author{
Sonia Otero- Estévez \\ Grupo de Investigación en Sociología de la Alimentación. Departamento de Sociología. Universidad de Oviedo \\ U0221461@uniovi.es \\ Jessica Paddock \\ University of Manchester \\ jessica.paddock@manchester.ac.uk331
}

"The practice of eating" es uno de los mayores esfuerzos que se han hecho por unificar una Teoría Sociológica de la Alimentación que responda a la compleja tarea de conocer cómo comemos. Al mismo tiempo, es una magnífica síntesis teórica y empírica de la práctica alimentaria y de su evolución a lo largo del tiempo en las sociedades contemporáneas.

El libro comienza en el primer capítulo recordando el creciente interés por las cuestiones relacionadas con la alimentación. Son muchas las disciplinas que han abordado la alimentación y los temas relacionados. Sin embargo, no ha sido hasta la pasada década de los años ochenta cuando los estudios de este tipo se intensificaron. A través de las páginas, "The practice of eating" va guiando al lector hacia la idea de que la alimentación reúne todas las características para ser analizada como una práctica social. La alimentación no es sólo un proceso fisiológico y ya desde las primeras páginas del libro puede observarse la intención del autor por mostrar la dimensión social y cultural del acto alimentario.

El principal desafío del autor en este libro es tratar de mostrar al lector que la alimentación es una práctica social compleja. La definición de un conjunto de conceptos le sirven para enmarcar el acto alimentario como un momento de consumo que juega un rol importante en la construcción de las identidades de los sujetos. Los objetivos que se plantea para llevar a cabo este propósito son tres. El primero de ellos es aportar claridad a la definición de "comer" y, al mismo tiempo, proponer un conjunto de conceptos para el análisis y la comprensión de la práctica alimentaria como una forma de consumo, 0 , dicho de otro modo, como una señal de identidad. El segundo es plantear el uso y mostrar el valor de las Teorías de la Práctica Social en la construcción de una teoría sociológica de la alimentación. Partiendo de la crítica y de las deficiencias de los análisis culturales, muestra cómo las Teorías de la Práctica Social se pueden aplicar a actividades altamente complejas, pero débilmente reguladas; como es el caso de la alimentación. Por último, destacar la importancia del estudio de la práctica alimentaria para comprender cómo gestionan los individuos la alimentación cotidiana en un escenario global cada vez más complejo.

El libro consta de ocho capítulos estructurados en tres partes. La primera parte, compuesta por los capítulos 2, 3 y 4, es una revisión teórica a través de la que se asientan las bases de sociología de la alimentación y que sirve de soporte para los capítulos sucesivos. Para esta primera parte el autor revisa las investigaciones en torno a la alimentación e introduce las teorías de la práctica social. La segunda parte, compuesta por los capítulos 5, 6 y 7 , se centra en el desarrollo de conceptos claves, como el de habituación y repetición, para el análisis de la alimentación como práctica social. Por último, en la tercera parte, el capítulo 8, se presenta una síntesis de los aspectos abordados a lo largo del libro y se ofrece un ejemplo ilustrativo de su aplicación. El libro evita hacer argumentos puramente teóricos y aporta en cada capítulo ejemplos prácticos.

En el segundo capítulo, se ilustran las raíces de los estudios alimentarios para poner de manifiesto 
la presencia a lo largo de la historia de los aspectos alimentarios en los estudios cientíicos y también para mostrar los obstáculos para la implementación de su síntesis teórica. En primer lugar, los estudios alimentarios han estado ligados tradicionalmente a dos campos: la nutrición y la agricultura. El primero de ellos focaliza en problemas alimentarios de carácter individual 0 nutricional. El segundo en las cuestiones relacionadas con la producción, y en las condiciones políticas en las que esta tiene lugar. Sin embargo, ambas se han olvidado de conectar estas cuestiones con el carácter más social y estructurante del acto alimentario. A pesar de años de tradición de estudios de consumo, las investigaciones sobre alimentación siguen mayoritariamente centradas en productos, producción y provisión. № se ha prestado atención a los aspectos más sociales y simbólicos de la alimentación: las comidas, las relaciones... etc. En segundo lugar, al ser un tema abordado desde muchas disciplinas, la síntesis teórica no ha sido vista como un aspecto prioritario. Y, en tercer lugar, ha sido observado siempre como un terreno plagado de cuestiones prácticas y en crisis.

El capítulo continúa haciendo un recorrido por las principales líneas temáticas en relación a la alimentación para mostrar cómo, en la actualidad, las preocupaciones y el contexto dónde la alimentación tiene lugar han cambiado. De ahí que los estudios alimentarios hayan ampliado sus líneas temáticas para preguntarse, entre otras cuestiones, por los peligros sociales de la moderna industria alimentaria, por la desestructuración alimentaria, por los peligros acerca de cómo comemos, o por cuestiones de consumo ético-político. Estos últimos aspectos han producido una importante colección de estudios que han situado de nuevo las cuestiones alimentarias en el centro de atención.

Finalmente, Warde cierra el capítulo centrándose en las cuatro principales corrientes en el estudio de la alimentación: análisis estructuralistas, análisis feministas, análisis sobre la relación entre la jerarquía social y los patrones de consumo de alimentos y, finalmente, el "Cultural Turn". Con el objeto de distanciarse de los modelos que enfatizan el individualismo y la racionalidad total del individuo han emergido otros enfoques. Entre ellos se encuentran las Teorías de la Práctica Social, que están siendo incorporadas en disciplinas muy diversas. Aquí es donde hará más hincapié Alan Warde en el tercer capítulo para demostrar la utilidad del uso de estas teorías en los análisis de la alimentación.

Las Teorías de la Práctica Social surgen como una explicación que pretende ir más allá de las aportaciones de la corriente funcional-estructuralista. Bourdieu y Giddens tendrán un papel clave dentro de esta teoría al centrar sus análisis en la teorización de las prácticas sociales, configurando lo que podría denominarse como la primera etapa dentro de las Teorías de la Práctica Social. Ambos otorgan un papel central a las prácticas sociales en el análisis de la interrelación entre individuo y estructura. A diferencia del paradigma funcionalista o estructuralista, esta teoría se aleja de la visión Durkheimiana totalizadora de la sociedad y otorga al sujeto un papel activo.

La segunda etapa de las Teorías de las Prácticas Sociales está encabeza por Schatzki y su libro The Practice Turn in Contemporary Theory (2001), escrito junto a Knorr Cetina y von Savigny. Su trabajo es un intento de superar los dos enfoques ontológicos dominantes: el individualismo y el holismo. Este autor aporta una nueva noción de prácticas al hacer distinción entre prácticas dispersas ("Dispersed practices") y prácticas integradoras ("Integrative practices"). Las primeras serían aquellas prácticas que requiere compresión y que hacen referencia a la capacidad de los individuos para seguir reglas, imaginarlas, explicarlas. Las segundas, mucho más complejas, serían reconocidas entre los miembros de una misma cultura sin ser explicadas, son aceptadas, tienen un vocabulario específico y se encuentran en las actuaciones y no en la mente de los individuos.

Después de guiar al lector hacía la idea de que las prácticas son la unidad fundamental de los análisis sociales, Warde se pregunta en el cuarto capítulo qué tipo de práctica social es la alimentación y qué elementos son claves para su análisis. Su objetivo es proporcionar al lector herramientas y algunas claves conceptuales que ayuden a enmarcar la práctica alimentaria y que recojan sus principales dimensiones: culinaria, corporal y social. 
Warde entiende que toda teoría de la alimentación necesita explicar la relación entre los alimentos que consumimos, el proceso de incorporación al cuerpo y la organización social en torno a la comida. Por eso, es importante definir de forma precisa algunos términos. En particular, se centra en los conceptos de evento y ocasión con los que aborda el significado social de la alimentación. La rutinización de la alimentación muestra un fuerte comportamiento colectivo en torno a la misma. También se detiene en los conceptos de comida, menú y plato para mostrar las diferencias culturales y sociales de las decisiones culinarias. Lo que comemos y lo que damos de comer a los otros depende en gran medida del contexto cultural y social. Y por último, se aproxima al concepto de incorporación con el que muestra la dimensión estético-afectiva de las preferencias alimentarias y las técnicas corporales que se aprenden y se ponen en práctica en cada contexto social para alimentarnos. Con estos conceptos se busca proporcionar un marco general de análisis que explique las diferencias alimentarias entre distintas poblaciones y que ponga énfasis en cómo y por qué las personas adoptan esas prácticas.

El capítulo cinco, que inicia la segunda parte del libro, trata de explicar cómo está organizada la alimentación. El capítulo parte de la idea de la existencia de una enorme variedad y variación en las prácticas alimentarias. Warde sugiere que este aspecto puede llevar a la reflexión errónea de que los individuos tienen un comportamiento desorganizado y que, por lo tanto, cualquier orden que pueda existir dentro de la práctica alimentaria se forma a partir de un tumulto de consumidores ansiosos e inciertos, sufriendo la condición que Fischler (1980) Ilamó gastro-anomie. Partiendo de esta idea, a lo largo del capítulo se rebate la explicación que reduce los logros de los individuos a una explicación de carácter individual. Warde regresa a la teoría de las prácticas sociales para mostrar cómo las actuaciones se someten a procesos de generación, organización y regulación colectiva. Las prácticas tienen normas que permiten instruir a otro en su desempeño exitoso. Los libros de cocina, los suplementos de periódicos y los programas de televisión sirven de buenos ejemplos para instruir hacia esa buena práctica.
Alan Warde llega a la conclusión, a partir de la división de Schatzki (véase capítulo 3), de que el acto alimentario no cumple todos los requisitos necesarios para ser definido como una práctica integrativa. Comer es una práctica demasiado compleja, y la instrucción sobre su desempeño se distribuye en muchos otros dominios que tienen sus propias prioridades; desde la nutrición y la cocina, hasta el gusto. Prioridades que a menudo pueden incluso competir entre sí. Este es el caso de los libros de cocina y las recomendaciones nutricionales. Mientras que los primeros son textos que instruyen en la mejora del sabor y gusto de los platos, los segundos dan recomendaciones sobre cómo deberíamos comer que no siempre son compatibles con la palatabilidad. Estas son algunas de las formas mediante las que una práctica se vuelve objetivada e institucionalizada, creando convenciones de comportamientos culinarios razonables.

Para explicar cómo es posible gestionar la alimentación, a pesar del caos de opciones posibles, el capítulo seis se adentra en los procesos de habituación y rutina. Siguiendo a Thaler y Sunstein (2009), Warde introduce la idea de que la práctica alimentaria no puede ser explicada por la aplicación consciente de las reglas, sino que más bien implica cierto grado de despreocupación y, sobre todo, automatización. Dicho de otro modo, hay un elemento "automático" en la práctica alimentaria que genera comportamientos constituidos por movimientos y acciones rápidas, inconscientes y hábiles. Los individuos a menudo no pueden explicar y expresar sus acciones cotidianas porque éstas se realizan intuitivamente y sin reflexión previa.

Para ilustrar la naturaleza irreflexiva de las actividades cotidianas, se basa en el ejemplo de un experimento realizado por Wansick (2006), en el que los sujetos se sirvieron y disfrutaron de un plato de sopa, tal como lo harían en un restaurante. Sin embargo, la mitad de los comensales tenían platos que se conectaban a un dispositivo que, poco a poco, rellenaba su tazón sin que ellos fueran conscientes. Estos comensales comieron un 73 por ciento más que sus homólogos, ofreciendo una explicación acerca de por qué la gente suele comer más allá de la saciedad. El apetito no está controlado por el pensamiento racional 0 la deliberación, sino 
que se guía mayoritariamente por la costumbre (por ejemplo, comiendo hasta que el plato esté vacío). De esta manera se recuerda que los diferentes contextos ofrecen un conjunto razonable o previsible de posibles comportamientos que se van incorporando a través de la repetición.

Sea cual sea la perspectiva desde la que se observe, Warde está convencido de que existe una relación más compleja entre lo social y lo individual de lo que sugiere el modelo de la elección individual. Para ilustrar esto, se apoya en la creciente prevalencia de la obesidad en occidente, y en el debate concomitante sobre sus causas y sus implicaciones. La explicación de la prevalencia de la obesidad se hace fundamentalmente a través de dos posturas. Por un lado, la de aquellos que consideran que la obesidad es resultado de una falta de voluntad por parte de los individuos para controlar su apetito y, en consecuencia, su peso corporal. Por otro lado, la de quienes atribuyen la responsabilidad a la existencia de un entorno social y económico que produce, promueve y vende alimentos que lo favorecen. Warde se apoya en estas explicaciones para mostrar que se trata de un problema que va más allá del individuo. De este modo, invita al lector a pensar en los factores que una explicación práctica-teórica de la obesidad debería enfatizar. Su intención no es otra que conducir hacia la reflexión de que - como en el experimento de la sopa de Wanwick - los hábitos incorporados, las rutinas temporales y las convenciones sociales son la clave en este asunto.

El capítulo siete reflexiona sobre el concepto de repetición y su utilidad para el análisis de las prácticas sociales. El énfasis en este concepto se hace, sobre todo, para explicar cómo dichas prácticas pueden organizarse y compartirse socialmente. Al inicio del capítulo, el autor afirma que las explicaciones sobre el hábito no son suficientes por si mismas para proporcionar un modelo general de acción. Se insta así a explorar el sentido práctico, es decir, la experiencia y el dominio de los procedimientos en la vida cotidiana. Para comprender mejor lo que se sabe sobre el hábito, Warde retoma las tesis de Bourdieu y su idea de las disposiciones que son centrales en la definición del habitus. Bourdieu consideraba que las disposiciones son fundamentales para el sentido práctico del juego que despliegan los actores sociales y que les guía, sin necesidad de reflexión previa, hacia la adopción de las acciones adecuadas. Las disposiciones dan un conjunto de respuestas, señales y pistas para la acción que no requieren reflexión in situ y que terminan por convertirse en rutina tras la propensión a repetir los mismos actos cuando se presentan las circunstancias apropiadas.

En la segunda parte del capítulo el autor introduce el concepto de rutina el cual implica la repetición temporalmente regular de una serie de acciones. Las rutinas añaden una dimensión adicional porque implican una acción más regularizada y pautada que el hábito, lo que las convierte en frecuentes y previsibles. Esto es importante, recuerda Warde, para comprender cómo se transmiten las habilidades. La gente aprende a través de la exposición y la repetición. Dichas rutinas pueden ser tanto personales como colectivas, como lo demuestran, por ejemplo, los patrones de horario de comida y los horarios de trabajo entre distintos países que a menudo comparten rasgos de organización y secuenciación.

Finalmente, en el capítulo ocho, se sintetizan las aportaciones que el autor ha ido realizando a lo largo del libro y se insiste en la utilidad que tienen la teoría de las prácticas sociales para la comprensión del acto alimentario. Alan Warde ha dedicado los últimos años de su investigación a profundizar en el análisis de la comida fuera del hogar. Esto le sirve para proporcionar un ejemplo sobre cómo la teoría de las prácticas sociales puede ser utilizada en los análisis alimentarios y cuál es el potencial de su uso. Siguiendo los datos publicados en el libro Eating Out: Social Differentiation, consumption and Pleasure (2000) que Alan Warde escribió con Lydia Martens, pone ejemplos para demostrar cómo los individuos comparten un conjunto de entendimientos y procedimientos comunes acerca de lo que supone alimentarse fuera del hogar.

Las razones para comer fuera de casa, aunque son varias, son compartidas. Y la inmensa mayoría ve la alimentación fuera del hogar como una actividad que le gusta. No obstante, como toda práctica social, la alimentación fuera del hogar no es vivida por todos de la misma forma. Existen diferencias 
en las actuaciones en función de la clase social, la etnia 0 el género. Cada grupo social comparte entendimientos, tiene procedimientos comunes, y un conjunto de convenciones que gobiernan y dirigen, alrededor de unas normas compartidas, la alimentación fuera del hogar (Warde y Martens, 2000). Trata de mostrar cómo el guión seguido para alimentarse fuera del hogar está predefinido incluso antes del propio acto y cómo mediante la repetición y rutinización de sus acciones van incorporando "dichos y hechos", van definiendo la práctica alimentaria.

En definitiva, The Practice of Eating guía al lector por los entresijos de la práctica alimentaria en las sociedades modernas. En esta obra se puede hacer un recorrido desde los inicios de la alimentación en los estudios científicos, hasta los avances actuales y sus nuevas temáticas. Quien lo lea tendrá un repertorio mucho más amplio de conceptos para abordar las cuestiones alimentarias y, si no lo hacía ya, habrá incrementado tanto las ganas como las razones para poner en marcha la compleja tarea de conocer cómo comemos. Precisamente por esta última razón, se hace necesario destacar también su valor didáctico que le confiere a este material una gran utilidad para quien se inicie en el estudio de la alimentación. Autores clásicos, ejemplos ilustrativos, definiciones precisas y, sobre todo, Sociología de la Alimentación es lo que se puede encontrar en "The practice of eating".

\section{BIBLIOGRAFÍA}

Fischler, C. (1980). Food habits, social change and the nature/culture dilemma. Information (International Social Science Council), 19 (6), 937-953.

Savigny, E. V., Knorr-Cetina, K., y Schatzki, T. R. (2001). The Practice Turn in contemporary theory. London: Routlegde.

Thaler, R. H., y Sunstein, C. R. (2009). Nudge: Wie man kluge Entscheidungen anstößt. Ullstein eBooks.

Wansink, B. (2006). Mindless eating: Why we eat more than we think. Bantam.

Warde, A., \& Martens, L. (2000). Eating out: Social differentiation, consumption and pleasure. Cambridge University Press. 
